\title{
Molecular Subtyping: status of the molecular factors in the locally advanced breast cancer and its correlation with risk factors.
}

\author{
Hasan Shahrear Ahmed, Krisna Rani Majumder, Mohammad Jahan Shams, AFM Anwar Hossain, \\ Md Mizanur Rahman
}

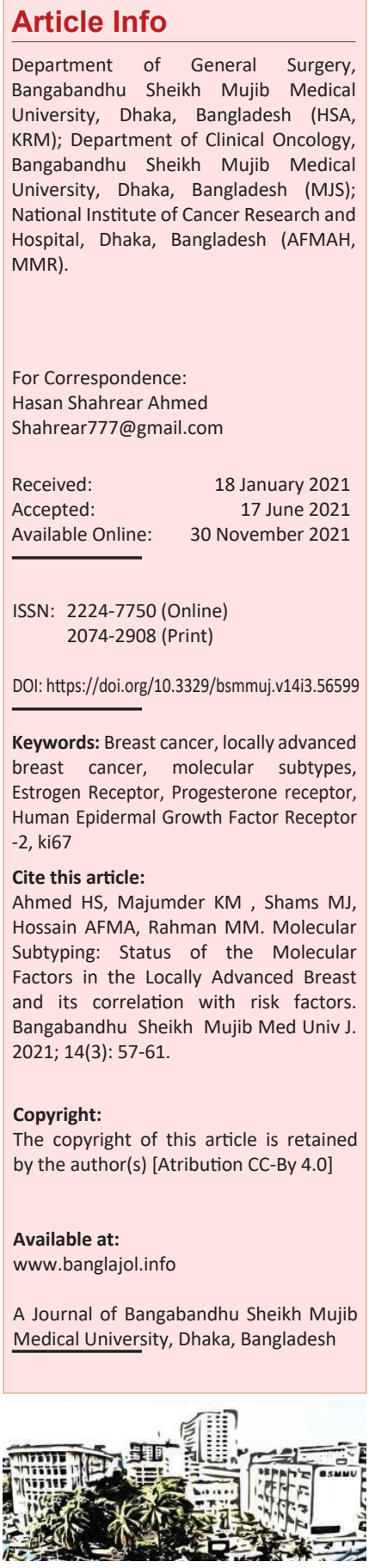

\begin{abstract}
Molecular factors play an important role in the management and treatment outcome of breast cancer. Molecular subtyping has been developed depending upon estrogen and progesterone receptors, human epidermal growth factor receptor-2 and ki76 level. This cross-sectional study was done to assess the molecular subtypes of locally advanced breast cancer and its associated risk factors. Total 94 patients with locally advanced breast cancer were included in the study. The mean age was 42.6 years with a standard deviation of 9.56 . In total, 91.5 percent of respondents had menarche at or after the age of 12 , and $26.6 \%$ had used hormonal contraception in the past. Tobacco users and positive family history were found in $21.2 \%$ and $5.35 \%$ of the cases. Among the patients, $3.4 \%$ of cases had their first childbirth after the age of 30 and $95.5 \%$ of patients feed their babies from their both breasts. Among 94 cases 5 did not have any child. Estrogen receptor was found positive in 35\% of cases, progesterone receptor-positive patient was 33\% and HER-2 was found positive in 39.4\% of cases. Ki-67 level was found high in $66 \%$ of cases. Among the 94 cases, the Luminal A subtype was found in $18 \%$ and the Luminal B subtype was found in $27.7 \%$ cases. The human epidermal growth factor receptor- 2 subtype was found relatively less frequent than Luminal type B (24.5\% vs. $27.7 \%)$. Triple-negative breast cancer was most commonly diagnosed among the patients (almost 30\%). The increased number of triple-negative variants signifies poor prognostic outcomes. The risk factor of breast cancer did not show any statistical correlation with molecular subtypes.
\end{abstract}

\section{Introduction}

Breast cancer is one of the major concerns in the realm of oncology as it is the most commonly occurring malignant disease in female with $23 \%$ of all new cases and $14 \%$ of all cancer-related deaths. 1 With the advancement of management protocol early detection, overall survival duration is increasing without any doubt. One notable phenomenon among the patients with breast cancer is that histologically similar type of diagnosis in the same stage of disease does not show the same prognosis with the similar treatment modality. It is largely due to the difference in its molecular factors. To date, several molecular factors have been identified that have shown different impacts on treatment and prognosis. Among those factors Estrogen and Progesterone Receptors (ER and PR), Human Epidermal Growth Factor Receptor (HER-2), TP53, Ki67, BRCA 1, BRCA 2, P 14 ARF (a locus with the multifunctional activity of tumour suppression), Cyclin D1 (cell cycle progression regulatory protein), Cycline $\mathrm{E}$, different cytokeratins (5/6), B Myb-a protooncogene, Twist (transcriptional repressor that effects E-Cadherin), DMP1 $\beta$, VEGF, TBX2/3 were found critical in various aspect of breast cancer management. $\frac{2,3}{3}$ Different molecular factors have been adopted to develop a surrogate definition of molecular subtypes in the St Gallen International Breast Cancer Conference in 2011 as Luminal A(ER and/or PR positive HER-2 negative and low ki67, Luminal B (ER and/or PR positive HER-2 positive and/ low ki67 or ER and/or PR positive HER-2 negative and high ki67, HER2 type(ER and PR negative, HER-2 positive) and tipple negative(TNBC) (ER, PR and HER-2negetive). $\frac{4.5}{~ T h e s e ~ s u b t y p e s ~ h a v e ~}$ shown a remarkable difference in treatment outcome. As this part of the globe still encountering a substantial number of locally advanced breast cancer (LABC) patients in comparison with the western world, $52.5 \%$ versus $7 \%$, pretreatment molecular subtyping 
of LABC will play an important role in overall management. $\underline{6}$ The subtyping has enlightened the heterogeneity of ER-positive tumours in treatment outcomes. The luminal A subtype has a favourable prognosis compared to the luminal B subtype and the systemic therapy advocated for the patients with luminal A tumour is generally restricted to endocrine therapy. The luminal B subtype has a high proliferation rate and/or a high histological grade and systemic treatment with chemotherapy followed by endocrine therapy is recommended. $\underline{4}$ 5. Human Epidermal Growth Factor Receptor Type 2 (HER2) has been used as predictive markers for identifying a high-risk phenotype and for the selection of the most efficient therapies. $\underline{7}$ The prognostic and predictive value of Ki-67 was evaluated in a review developed by Luporsi et al, 2012 and they concluded that this biomarker could be considered as a prognostic factor for the therapeutic decision. $\underline{8}$

This study was performed to observe molecular subtyping in locally advanced breast cancer and its association with known risk factors of breast cancer like age, the onset of menarche, hormonal contraceptive use, menopause, tobacco consumption, and family history, age of $1^{\text {st }}$ childbirth, and breastfeeding practice.

\section{Materials and Methods}

This cross-sectional study was conducted from July 2014 to June 2016. According to inclusion and exclusion criteria, patients were enrolled in the study after physical examination and investigation. A total of 94 patients with locally advanced breast cancer (T3N0/T2N2/T3N1-2/T4Nx/TxN3) attending the outpatient and inpatient departments of the National Institute of Cancer Research and Hospital (NICRH), Mohakhali, Dhaka were included. Ethical clearance was obtained from the Ethical Committee of NICRH. A structured pre-tested questionnaire containing patient profiles was prepared. Informed written consent was obtained from each patient. As gene expression array data is not routinely available immunohistochemical (IHC) marker, expression data was used for molecular subtyping i.e. luminal A (ER + and/or PR+, Ki67 low and HER2-), luminal B (ER + and/or PR+, Ki-67 high and/ or HER2+), HER2-positive (ER-, PR- and HER2+) and triple-negative (ER-, PR-, and HER2-). 4 The collected paraffin blocks prepared from core cut biopsy and mastectomy specimen from Histopathology department of NICRH were sent for immunohistochemistry for ER, PR, HER-2 and Ki67. HRP (Horseradish Peroxidase) polymer-based detection system was used for all immunohistochemistry. Allred scoring method was used for ER, PR expression in breast cancers, scores 0 - 2 are deemed negative while scores 3-8 denote positive expression (Hammond ME et al 2010). HER-2 scoring was done as per the ASCO/CAP reconciled guidelines for HER-2/neu expression in breast cancer. ${ }^{9} \mathrm{Ki}-67$ values were acquired as the percentage of positively marking malignant cells using the anti-human Ki-67 monoclonal antibody MIB1 which is one of the most commonly used antibodies and is considered as the gold standard. ${ }^{10}$ Reports were collected and recorded in the data collection sheet. A purposive sampling technique was applied. For analysis of the data, Statistical Package for Social Sciences (SPSS) for Windows (IBM SPSS Statistics for Windows, version 23.0) was used. To see the association between categorical variables Chi-Square test (and Fisher's exact test where applicable) was performed. A $p$-value $\leq .05$ was considered statistically significant.

\section{Results}

In this study, 94 patients with diagnosed locally advanced breast cancer were included. The mean (SD) age of the patients was 42.6 (9.56) years (Figure-1). More than $50 \%$ patient was in the 40 to 60 years age group. Only 2 patients were found in the 61 to 80 years age group (Table - I).

Most of the patients had menarche $\geq 12$ years of age $(91.5 \%)$ and $26.6 \%$ had a history of using hormonal contraceptives. Tobacco users and positive family history were found in $21.2 \%$ and $5.35 \%$ of the cases. Menopause was found in only $10(10.6 \%)$ of cases

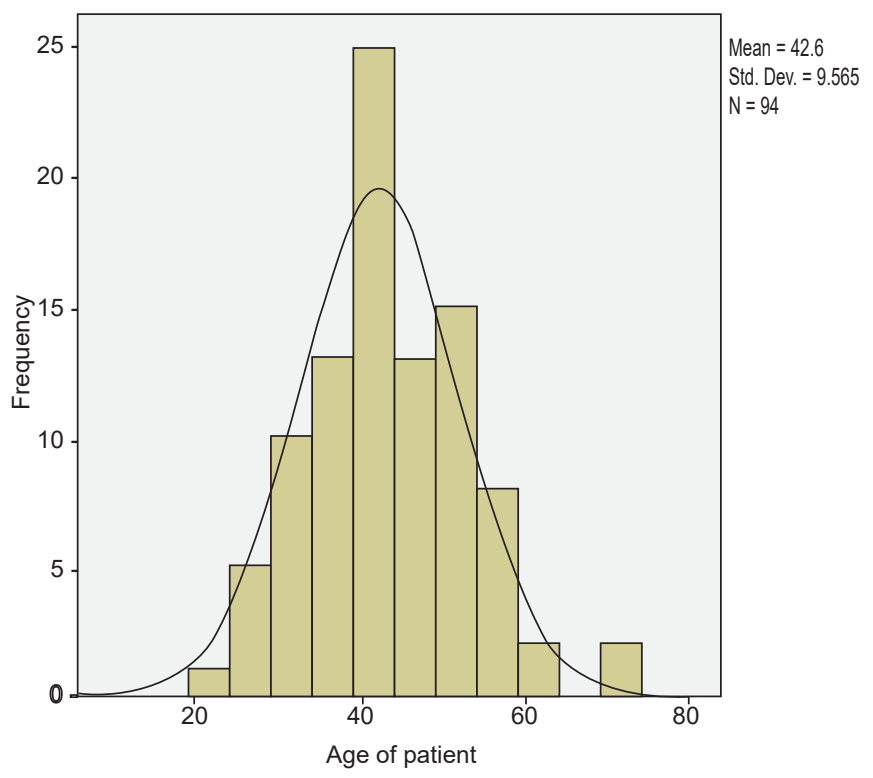

Figure - 1: Histogram showing age distribution of the patients

\section{Table-I}

Distribution of patients by age group ( $n=94)$

\begin{tabular}{|c|c|c|c|}
\hline \multicolumn{1}{|c|}{ Variable } & Data & Frequency & Percentage \\
\hline Age group (years) & $20-40$ & 44 & 46.8 \\
\cline { 2 - 4 } & $41-60$ & 48 & 51.1 \\
\cline { 2 - 4 } & $61-80$ & 2 & 2.1 \\
\hline
\end{tabular}


(Table - II). Among the patients, 3.4\% had their first childbirth after the age of 30 and $95.5 \%$ fed their babies from both breasts (Table - III). Total 5 cases did not have any child, 2 were unmarried. ER was found positive in $35 \%$ of cases, PR positive patient was 31 in number and HER2 was found positive in $39.4 \%$ of cases. In $66 \%$ of the cases were with $\mathrm{Ki}-67$ level $>14 \%$ in the highest proliferative area (Table - IV).

\section{Table-II}

\section{Distribution of patients by risk factors $(n=94)$}

\begin{tabular}{|l|c|c|c|}
\hline \multicolumn{1}{|c|}{ Variable } & Data & Frequency & Percentage \\
\hline \multirow{3}{*}{ Onset of Menarche } & $<12$ years & 8 & 8.5 \\
\cline { 2 - 4 } & $\geq 12$ years & 86 & 91.5 \\
\hline \multirow{2}{*}{ Hormonal } & Yes & 25 & 26.6 \\
\cline { 2 - 4 } contraceptive use & No & 69 & 73.4 \\
\hline Menopause & Yes & 15 & 16.0 \\
\cline { 2 - 4 } & No & 79 & 84.0 \\
\hline Tobacco & Yes & 20 & 21.3 \\
\cline { 2 - 4 } consumption & No & 74 & 78.7 \\
\hline Family history & Positive & 5 & 5.3 \\
\cline { 2 - 4 } & Negative & 89 & 94.7 \\
\hline
\end{tabular}

Table-III

Distribution of patients by Maternity and Breastfeeding $(n=89)$

\begin{tabular}{|l|c|c|c|}
\hline \multicolumn{1}{|c|}{ Variable } & Data & Frequency & Percentage \\
\hline \multirow{3}{*}{ Age of 1st childbirth } & s30years & 86 & 96.6 \\
\cline { 2 - 4 } & $>30$ years & 3 & 3.4 \\
\hline \multirow{2}{*}{ Breast Feeding } & Yes & 85 & 95.5 \\
\cline { 2 - 4 } & No & 4 & 4.5 \\
\hline
\end{tabular}

Among the 94 cases, the Luminal A subtype was found in $15(18 \%)$ and the Luminal B subtype was found in $26(27.7 \%)$ cases. HER-2 subtype was found relatively less frequent than Luminal type B $(24.5 \%$ vs. $27.7 \%)$. The important phenomenon is that TNBC (triple-negative), which is the clinically most aggressive variant was diagnosed in maximum (almost 30\%) patients (Table-V). This information plays a definitive concern in the management plan. No statistical association was found between the molecular subtyping and the risk factors of breast cancer (Table -VI).

\section{Table-IV}

Distribution of patients by molecular factors $(n=94)$

\begin{tabular}{|l|c|c|c|}
\hline Variable & Data & Frequency & Percentage \\
\hline Molecular factors & Positive & 33 & 35.1 \\
\cline { 2 - 4 } Estrogen Receptor (ER) & Negative & 61 & 64.9 \\
\hline \multirow{2}{*}{$\begin{array}{l}\text { Progesterone } \\
\text { Receptor (PR) }\end{array}$} & Positive & 31 & 33.0 \\
\cline { 2 - 4 } & Negative & 63 & 67.0 \\
\hline \multirow{2}{*}{ HER-2 } & Positive & 37 & 39.4 \\
\cline { 2 - 4 } & Negative & 57 & 60.6 \\
\hline \multirow{2}{*}{ Ki-67 percentage } & $\leq 14 \%$ & 32 & 34.0 \\
\cline { 2 - 4 } & $>14 \%$ & 62 & 66.0 \\
\hline
\end{tabular}

Table-V

Distribution of patients by molecular subtypes

\begin{tabular}{|l|c|c|c|}
\hline Variable & Data & Frequency & Percentage \\
\hline Molecular subtypes & Luminal A & 17 & 18.1 \\
\cline { 2 - 4 } & Luminal B & 26 & 27.7 \\
\hline \multirow{5}{*}{} & HER-2 & 23 & 24.5 \\
\cline { 2 - 4 } & TNBC & 28 & 29.8 \\
\hline
\end{tabular}

Table-VI

\section{Relationship between molecular subtypes and risk factors}

\begin{tabular}{|c|c|c|c|c|c|c|c|}
\hline Variables & Data & Luminal n (\%) A & Luminal B n (\%) & HER2 n (\%) & TNBC n $(\%)$ & Total n (\%) & P-value \\
\hline \multirow[t]{2}{*}{ Age (years) } & $\leq 40$ & $11(11.7)$ & $11(11.7)$ & $13(13.8)$ & $9(9.2)$ & $44(46.8)$ & \multirow[t]{2}{*}{.53} \\
\hline & $>40$ & $10(10.6)$ & 15 (15.9) & $10(10.6)$ & $15(15.9)$ & $50(53.2)$ & \\
\hline \multirow[t]{2}{*}{ BMI } & $\leq 24.9$ & $13(13.8)$ & $23(24.4)$ & $21(22.3)$ & $23(24.4)$ & $80(85.1)$ & \multirow[t]{2}{*}{.60} \\
\hline & $>25.0$ & $4(4.2)$ & $3(3.2)$ & $2(2.1)$ & $5(5.3)$ & $14(14.9)$ & \\
\hline \multirow[t]{2}{*}{ Menarche (years) } & $<12$ & $1(1.1)$ & $2(2.1)$ & $4(4.2)$ & $1(1.1)$ & $8(8.5)$ & \multirow[t]{2}{*}{$.39 *$} \\
\hline & $\geq 12$ & $16(17.0)$ & $24(25.5)$ & $19(20.2)$ & $27(28.7)$ & $86(91.4)$ & \\
\hline \multirow[t]{2}{*}{ Breast feeding } & Yes & $17(18.0)$ & $24(25.5)$ & $22(23.4)$ & $27(28.7)$ & 90 ( 95.7) & \multirow[t]{2}{*}{$.83^{*}$} \\
\hline & No & $0(0)$ & $2(2.1)$ & $1(1.1)$ & $1(1.1)$ & $4(4.2)$ & \\
\hline \multirow{2}{*}{$\begin{array}{l}\text { Hormonal } \\
\text { contraceptive use }\end{array}$} & Yes & $5(5.3)$ & $5(5.3)$ & $7(7.4)$ & $8(8.5)$ & $25(26.6)$ & \multirow[t]{2}{*}{.79} \\
\hline & No & $12(12.7)$ & $21(22.3)$ & $16(17)$ & $20(21.2)$ & $69(73.4)$ & \\
\hline \multirow[t]{2}{*}{ Menopause } & Yes & $2(2.1)$ & $4(4.2)$ & $2(2.1)$ & $7(7.5)$ & $15(15.9)$ & \multirow[t]{2}{*}{$.48^{*}$} \\
\hline & No & $15(15.9)$ & $22(23.4)$ & $21(22.3)$ & $21(22.3)$ & $79(84.1)$ & \\
\hline
\end{tabular}

*Fisher's Exact Test 


\section{Discussion}

In this study, most of the patients were in the $41-60$ years age group followed by 20 - 40 years age group. No patient was found below 20 or above 80 years of age. The mean age of the patients was 42.6 years. A study by Rahman M 2015 showed a mean age of 44.7 years which was similar to this study. ${ }^{6}$

The present study showed that most of the patients $(91.5 \%)$ had menarche at or above 12 years of age and $26.6 \%$ had a history of using hormonal contraceptives. Jabeen $S$ et al observed that $54 \%$ of patients had menarche at eleven or below the age and $42 \%$ of patients used oral contraceptives. ${ }^{11}$ In an analysis of data from a multicenter, population-based case-control study, Marchbanks PA et al found that breast cancer risk did not vary by oral contraceptive use. ${ }^{12}$

Among the patients, tobacco users and positive family history were found in $21.2 \%$ and $5.35 \%$ of the cases. Jabeen S et al, 2013 reported in her study that majorities $(97 \%)$ of the respondents were nonsmoker and only $3 \%$ was a smoker. ${ }^{11}$ Epidemiological investigations of the relations between smoking and breast cancer have yielded conflicting results. Several studies have suggested that smoking may decrease the risk of breast cancer. Others have reported no evident association, while a few have suggested that smoking may increase the risk of breast cancer, especially in pre-menopausal women. ${ }^{13}$ A study by Anderson TI 1996 showed that about 20 $\%$ of breast cancer patients have a family history of the disease in a first-degree relative. ${ }^{14}$ In this study, menopause was found in only $15(16 \%)$ of cases which were $57.2 \%$ in a study conducted by Rahman M 2015. ${ }^{6}$ This discrepancy might be due to the inclusion of all stages of breast cancer in their study.

The study finding showed that about $97 \%$ of cases had their first childbirth below the age of 30 and $95.5 \%$ of patients feed their babies from both breasts. Most of the cases in this study were were housewives $(86.2 \%)$ and the office staff was foundto be $5.3 \%$. This was consistent with other studies. ${ }^{11,15}$ Palmer JR et al showed that a longer duration of breastfeeding has been associated with a greater reduction in breast cancer risk. ${ }^{16}$

Estrogen Receptor (ER) was found positive in 35\% of cases. Progesterone Receptor (PR) positive patient was 31 in number and HER-2 was found positive in $39.4 \%$ of cases. In $66 \%$ of the cases were with Ki-67 level $>14 \%$ in the highest proliferative area. Miglietta L et al, 2009 observed that ER receptors were low or absent in $29 \%$ and high in $71 \%$ of the tumors, PR receptors low or absent in $46 \%$ and positive in $54 \% .{ }^{17}$

TNBC subtype was found in approximately 28 (30\%) cases followed by Luminal B subtype $26(27.7 \%)$. Luminal A was found in 17 (18.1\%) cases. Wang J et al, 2016 reported in their study that there were $61(25.4 \%)$ with luminal A, 127(52.9\%) with luminal B, 31 (12.6\%) with HER-2 overexpression and $21(8.8 \%)$ were triple-negative type. $\frac{18}{}$ In another study by Prat A et al, 2015 observed that Luminal A, Luminal B and HER-2 types represented $30.6 \%, 18.2 \%$ and $10.3 \%$ respectively. $\underline{19}$ As all the recorded cases were selected with locally advanced breast cancer it can be assumed that aggressive variants will be common among the cases.

Regarding correlation among various risk factors and molecular subtypes, no statistical significance was found. Wang et al showed that menopausal status had an association between molecular subtypes. ${ }^{16}$ Turkoz et al found a significant association of age ( $\geq 40$ years), first baby at $\geq 30$ years, postmenopausal status and obesity with molecular subtypes; and no correlation with early menarche, late menopause, family history, oral contraceptive and smoking. ${ }^{20}$

\section{Conclusion}

The triple-negative subtype which is the most aggressive variant concerning prognosis was found as the most common subtype and the known risk factors of breast cancer did not show any association with a particular molecular subtype.

\section{Conflict of Interest}

The author declares no conflict of interest.

\section{References}

1. Jemal A, Bray F, Center MM, Ferlay J, Ward E, Forman D. Global cancer statistics. CA Cancer J Clin. 2011; 61:69-90.

2. Taneja P, Maglic D, Kai F, Zhu S, Robert D, Elizabeth A et al. Classical and Novel Prognostic Markers for Breast Cancer and their Clinical Significance. Clinical Medicine Insights: Oncology. 2010;4:15-34.

3. Inoue K, Fry EA. Novel Molecular Markers for Breast Cancer. Biomarkers in Cancer. 2016;8:25-42.

4. Goldhirsch A, Wood WC, Coates AS, Gelber RD, Thürlimann B, Senn HJ et al. Strategies for subtypesdealing with the diversity of breast cancer: highlights of the St Gallen International Expert Consensus on the Primary Therapy of Early Breast Cancer. Ann Oncol.2011;23:2997-3006.

5. Brouckaert O, Laenen A, Vanderhaegen J, Wildiers $H$, Leunen K, Amant F et al. Applying the 2011 St Gallen panel of prognostic markers on a large single hospital cohort of consecutively treated primary operable breast cancers. Ann Oncol. 2012;23(10):2578-2584.

6. Rahman M, Ahsan A, Begum F, Rahman K. Epidemiology, Risk Factors and Tumor Profiles of Breast Cancer in Bangladeshi underprivileged women. Gulf J Oncolog. 2015;1(17):34-42.

7. Weigelt B, Reis-Filho J S. Molecular profiling currently offers no more than tumour morphology and basic 
immunohistochemistry. Breast Cancer Research. 2010; 12(4): S5.

8. Luporsi E, Andre F, Spyratos F, Martin PM, Jacquemier J, Penault-Liorca F et al. Ki-67: level of evidence And methodological considerations for its role in the clinical management of breast cancer: analytical and critical review. Breast Cancer Research and Treatment. 2012;132(3):895- 915.

9. Wolff AC, Hammond MEH, Hicks DG, Dowsett M, McShane LM, Allison $\mathrm{KH}$ et al. Recommendations for Human Epidermal Growth Factor Receptor 2 Testing in Breast Cancer. Arch Pathol Lab Med. 2014;138(2):241-256.

10. Dowsett M, Nielsen TO, A'Hern R, Bartlett J, Coombes RC, Cuzick J et al. Assessment of Ki-67 in breast cancer: recommendations from the International Ki67 in Breast Cancer Working Group. J Natl Cancer Inst. 2011;103:1656-1664.

11. Jabeen S, Haque M, Islam J, Hossain MZ, Begum A, Kashem MA et al. Breast cancer and some epidemiological factors: a hospital based study. J Dhaka Med Coll. 2013; 22(1) : 61-66.

12. Marchbanks PA, Curtis KM, Mandel MG, Wilson HG, Jeng $\mathrm{G}$, Folger SG et al. Oral contraceptive formulation and risk of breast cancer. Contraception. 2012;85:342-350.

13. Rohan TE, Baron JA. Cigarette smoking and breast cancer. Am J Epidemiol. 1989; 129:36-42.

14. Anderson TI. Genetic heterogeneity in breast cancer susceptibility. Acta Oncol. 1996;35:407-410.
15. Abou-Daoud KT. Cancer of the breast and breastfeeding: study of 279 parous women and matched controls. Cancer. 1971;32: 67-9.

16. Palmer JR, Boggs DA, Wise LA, Ambrosone CB, Adams-Campbell LL, Rosenberg $\mathrm{L}$ et al. Parity and lactation in relation to estrogen receptor negative breast cancer in African American women. Cancer Epidemiol Biomarkers Prev. 2011;20:1883-1891.

17. Miglietta L, Vanell P, Canobbio L, Parodi MA, Guglielmini $\mathrm{P}$, Boccardo F. Clinical and pathological response to primary chemotherapy in patients with locally advanced breast cancer grouped according to hormonal receptors, her2 status, grading and ki-67 proliferation index. Anticancer Research. 2009;29(5):1621-1626.

18. Wang J, Sang D, Xu B, Yuan P, Ma F, Luo Y et al. Value of Breast Cancer Molecular Subtypes and Ki67 Expression for the Prediction of Efficacy and Prognosis of Neoadjuvant Chemotherapy in a Chinese Population. Medicine. 2016; 95(18): 3518.

19. Prat A, Fan C, Fernandez A, Katherine A, Martinelo R, Vidal $M$ et al. Response and survival of breast cancer intrinsic subtypes following multi-agent neoadjuvant chemotherapy. BMC Medicine. 2015;13:303.

20. Turkoz FP, Solak M, Petekkaya I, Keskin O, Kertmen N, Sarici $\mathrm{F}$ et al. Association between common risk factors and molecular subtypes in breast cancer patients. Breast. 2013;22(3):344-50. 